\title{
Severe acute respiratory syndrome coronavirus-2 and pulmonary tuberculosis: convergence can be fatal
}

\author{
Abhijeet Singh ${ }^{1}$, Rajendra Prasad ${ }^{2}$, Ayush Gupta ${ }^{1}$, Kamanasish Das ${ }^{3}$, Nikhil Gupta ${ }^{4}$ \\ ${ }^{1}$ Department of Pulmonary and Critical Care Medicine, Medeor JCS Institute of Pulmonary, Critical Care and Sleep \\ Medicine, New Delhi; ${ }^{2}$ Director of Medical Education \& Head Pulmonary Medicine, Era's Lucknow Medical College \\ and Hospital, Lucknow; ${ }^{3}$ Department of Pulmonary and Critical Care Medicine, Ayushman Hospital and Health \\ Service, New Delhi; ${ }^{4}$ Department of General Medicine, Dr. Ram Manohar Lohia Institute of Medical Sciences, \\ Lucknow, India
}

\begin{abstract}
Disease associated with SARS-CoV-2 also termed as Coronavirus disease 2019 or COVID-19, has become a potential threat to public health by spreading across more than 200 countries worldwide within a short span of time. Tuberculosis (TB) is already existing as unprecedented pandemic worldwide over several years. Both diseases have many overlapping features but there are striking differences too. There is usually chronicity of symptoms in TB as compared to acute or rapid progression in COVID-19. Little evidence exists regarding TB and COVID-19 coinfection. It is anticipated that person with TB either in active, previously treated or latent forms are more at risk of poor outcomes with COVID-19. The relationship between the two diseases is still unclear at present, and
\end{abstract}

Correspondence: Dr. Abhijeet Singh, Department of Pulmonary and Critical care medicine, Medeor JCS institute of Pulmonary, Critical Care and Sleep Medicine, B-33, 34 Qutab Institutional Area, New Delhi 110016, India.

Tel. +91.8860872269 .

E-mail: appu.abhijeet @ gmail.com

Key words: COVID-19; SARS-CoV-2; tuberculosis; coinfection.

Conflict of interest: The authors declare no conflict of interest.

Contributions: AS, RP, AG, KD and NG made substantial contributions to the conception or design of the work; AS, AG, KD interpreted data; AS, AG, KD, NG have drafted the work or substantively revised it. All the authors have read and approved the final version of the manuscript and agreed to be accountable for all aspects of the work.

Funding: None.

Received for publication: 10 May 2020.

Accepted for publication: 13 July 2020.

CCopyright: the Author(s), 2020

Licensee PAGEPress, Italy

Monaldi Archives for Chest Disease 2020; 90:1368

doi: 10.4081/monaldi.2020.1368

This article is distributed under the terms of the Creative Commons Attribution Noncommercial License (by-nc 4.0) which permits any noncommercial use, distribution, and reproduction in any medium, provided the original author(s) and source are credited. more studies are needed to enable analyses of interactions and determinants of outcomes in patients affected by both the diseases. Most of the countries across the world imposed nationwide lockdown to promote social distancing, which is one important preventive measure to mitigate the spread of COVID-19 pandemic. However, it becomes quite challenging to ensure smooth functioning of programmatic services, leading to disruption of routine TB care, leading to transmission of infection. Health authorities should frame polices that can support TB patients by providing diagnostic, management and prevention services without any interruption during this era of ongoing COVID-19 pandemic. Effort should be made to control both the diseases simultaneously and avoid unfavourable outcome in near future.

\section{Introduction}

The 2019 novel coronavirus (2019-nCoV), recently renamed Severe Acute Respiratory Syndrome Coronavirus 2 (SARS-CoV2) by World Health Organization (WHO), was first notified from Wuhan City of Hubei Province of China in December 2019 in the form of unexplained pneumonia [1]. Disease associated with SARS-CoV-2 also termed as Coronavirus disease 2019 or COVID-19 is evolving rapidly and has been declared as a pandemic by WHO on $11^{\text {th }}$ March 2020. It has now become a potential threat to public health in a short span of time by affecting more than 200 countries worldwide with relevant impact on morbidity and mortality [1,2]. Tuberculosis (TB) is already existing as unprecedented pandemic worldwide over several years. It was already declared a global health emergency by WHO in 1993 [3]. The estimated global burden of TB is 10 million with mortality of 1.2 million among HIV-negative, and an additional 0.25 million among HIV positive people [4]. Nearly half of burden had drug resistance in 2018. South Africa and India carry the majority of global burden of TB and HIV coinfection. Out of 10 million cases, 3 million (30\%) remain undiagnosed. Around 30,000 people fall ill and 4000 die every day, despite the fact that this disease is preventable and curable. Around $25 \%$ of world population is also estimated to have latent TB infection (LTBI) [4]. There is significant heterogeneity in epidemiological profile of both diseases across all countries worldwide. The reasons responsible for this are difference in demographic profile, surveillance monitoring and policies adopted for delivery of health care depending upon existing infrastructure and economic feasibility. Therefore, the magnitude of burden regarding convergence of pandemics of TB and 
novel COVID-19 cannot be estimated at this moment. Very limited evidence exist regarding relationship between TB and COVID-19 till now. Therefore, the aim of this narrative review is to reflect latest update on interactions between TB and COVID-19.

\section{Search strategy}

A systematic search strategy was adopted involving principal electronic databases (PubMed, EMBASE, Web of Science, ISI Web of Knowledge, Google and Google scholar, Researchgate, COVID-19 literature search database) and documents reports/preprints by WHO, IUATLD, bioRxiv and medRxiv. Articles in English language from 2000 till now, were identified using the terms in combination: ["COVID" OR "COVID-19" OR "SARSCoV-2" OR "2019-nCoV" OR "Novel coronavirus"] AND ["SARS" OR "SARS-CoV" OR "SARS-CoV-1"] AND ["MERS" OR "MERS-CoV"] AND ["Tuberculosis" OR "TB" OR "Mycobacterium tuberculosis"] AND ["Association" OR "Coinfection" OR "Coexistence" OR "Interaction" OR "Relationship"]. Manual search was also performed in addition. Overall, 44 articles relevant to research topic were identified. All articles with resulting titles, abstract and full text, when available, were read and kept for references.

\section{TB and COVID-19: similarities and differences}

Although COVID-19 and TB share many similarities, there are also striking differences [5-7]. The causative agent for TB is bacteria Mycobacterium tuberculosis, whereas for COVID-19 is novel coronavirus SARS-CoV-2. Both can manifest with similar respiratory symptoms like fever, cough, breathlessness and weakness with varying severity, but there is chronicity of symptoms in TB as compared to acute or rapid progression in COVID-19. Both are transmitted mainly through close contacts. TB is primarily transmitted through droplet nuclei of aerosols generated by infected person with TB, who may be infectious for usually longer duration before initiation of effective treatment [8]. SARS-CoV-2 can transmit mainly through droplets and fomites, but can be transmitter through aerosols based on recent evidence [9]. The incubation period from exposure to disease in TB is usually longer, with slow onset and existing in active $(10 \%)$ or latent form depending upon the immune status [3,10] whereas COVID-19 may appear 2-14 days after exposure with median incubation period of five days and latent period is not defined [11]. COVID-19 can present with a variety of clinical presentations, asymptomatic to mild being most common (80\%), severe (14\%) and critically ill $(6 \%)$ [8,11,12]. A person, once infected with SARS-CoV-2, can transmit infection to at least 2 persons [12], but a person infected with TB can infect additional 1 to 4 persons [10]. Few risk factors are overlapping between TB and COVID-19 such as advanced age, male, diabetes, smoking, chronic kidney disease and other chronic respiratory illnesses. Younger age group, immunosuppressed conditions including HIV, malnutrition and chronic alcohol intake are proven risk factors for TB [10] whereas hypertension, obesity and cardiac disorders are related to COVID-19 [8]. More evidence is required to study association of various risk factors particularly HIV and TB with COVID-19. Concern is for pediatric population, who are already neglected for TB care and if not treated for COVID-19 pandemic, it can lead to poor outcome. Atypical symptoms for TB as well as COVID-19 in children might mislead diagnosis. TB is having case fatality rate (CFR) of $15 \%$ [4] as compared to COVID19 having CFR of $1.4-3.4 \%$ although estimation of exact values are unreliable at this point of time with spreading novel SARSCoV-2 infection [13]. However, we do have well defined management plans for TB including appropriate regimens, chemoprophylaxis, infection control and vaccines, but there is no specified treatment and vaccines apart from infection control for SARS-CoV-2 currently. Surveillance for TB is well defined but for SARS-CoV2 it is evolving and changing depending on the level of transmission. Initial testing was confined to those with a history of international travel to affected areas in last 14 days [14]. Active surveillance has been adopted once local or community transmission has become an issue, as testing has been expanded to patients with severe pneumonia of unknown cause that does not respond to antibiotics, symptomatic contacts of laboratory confirmed cases, symptomatic health care workers, asymptomatic direct and high risk contacts of a confirmed case between fifth and fourteenth day of contact and all symptomatic individuals with influenza like illness in hotspot areas or large migration gatherings [15]. Appropriate surveillance systems have been adopted for COVID19 within short span of time, whereas it still not optimized for more deadly TB due to poor funding. Phenomenon of reactivation and re-infection have been well established in previously treated cases of TB, but whether this is applicable for patients recovered from novel COVID-19 needs to be determined.

Diagnosis for pulmonary TB usually include sputum or bronchoalveolar lavage (BAL) staining for TB bacilli, phenotypic culture methods and rapid diagnostic tests like automated cartridge based nucleic acid amplification test (CBNAAT) like GeneXpert [4] whereas for COVID-19 require test like RT-PCR either from a nasal and oral pharyngeal swab or a sputum / BAL test if required [16]. CBNAAT can also be utilized for detecting SARS-CoV-2 infection. These tests can detect infection rapidly with better diagnostic accuracy. Radiological imaging like chest X-ray and computed tomographic imaging may be required to further refine the diagnosis and/or establish the severity of the disease (extent of ground glassing, patchy consolidation and interstitial abnormalities). TB usually presents with radiological features like parenchymal disease- unilobar or multilobar with or without cavitation, lymphadenopathy, pleural effusion and also endobronchial involvement with tree in bud opacities. But there can be overlapping or mixed patterns in case of coinfection.

Both disease rely on contact tracing and infection control measures such as hand hygiene, social distancing, regular cleaning of surfaces, triaging, cough etiquette, isolation, prolonged quarantine, ventilation and respiratory protection with barriers like masks and gloves [6,7]. Additional measures for TB include prompt treatment of active or latent form of TB rendering patient non-infective. Contact tracing and infection control measures have been aggressively reinforced to prevent novel COVID-19 since uncertainty exists regarding treatment and vaccination. These measures have also been adopted for TB control since many years, but still remains under-utilized as options of definitive treatment and chemoprophylaxis are available to act upon. Both diseases are associated with social stigma and has caused huge economic impact worldwide. However, the fear, apprehension and myths are more associated with novel COVID-19 among community and health care workers because of the uncertainty due to lack of scientific knowledge and awareness. Also the burden of disease remains acute as compared to chronic for TB. Therefore, national policies have been adopted rapidly in several countries whereas consistent policies are still lacking for TB $[6,7]$. 


\section{Relationship between tuberculosis and COVID-19}

Co-infection of TB with other coronaviruses like SARS-CoV1 and MERS-COV have been reported during outbreaks in 2003 and 2012 respectively [17-20]. All these cases were either having pulmonary TB initially followed by viral superinfection $[17,18,20]$ or vice versa [19]. The possible reason for such association could be that both diseases augment each other with transient decrease in cellular immunity leading to new infection or exaggerated reactivation of latent infection. This association of TB even with novel SARS-CoV-2 could not be underestimated based on the experience of previous coronaviruses. Evidence is limited whether person with TB whether latent, active or previously treated forms, are more at risk of poor outcomes with COVID-19. Post-tubercular sequelae can exist in the form of obstructive, restrictive or mixed airway impairment leading to chronic respiratory failure with or without features of cor-pulmonale. This residual damage to lungs can be a probable risk factor for developing more severe COVID19 symptoms and complications. Additional risk factors such as older age, comorbid illnesses, drug resistance and HIV coinfection will make situation more complicated and high chances of transmission of infection in community and mortality if left untreated. Recently, few studies have shown evidence on these anticipations to some extent, as described in Table 1 [21-27]. First ever study on cohort of 49 patients from 8 countries in 3 continents reflected that COVID-19 can be diagnosed before, simultaneously or after TB (pulmonary or extra-pulmonary) [21]. Mortality was higher in patients with post TB sequelae due to older age group and associated comorbidities. Drug resistance and HIV coinfection were also associated with few cases. The second study described clinical, radiological, and laboratory characteristics of a series of 20 COVID-19 patients with concurrent active TB in a hospital in northern Italy [22]. The third study described the characteristic of mortality in these combined cohorts of patients ( 49 patients from the first study and 20 patients from the second study) [26]. Mortality was more prevalent in elderly patients with co-morbidities and TB might not be a major determinant of mortality. Migrants from the cohort reported lower mortality rate than nonmigrants $(1 / 43,2.3 \%$ vs $7 / 26,26.9 \%)$ due to younger age and lesser number of co-morbidities in the former. Although it has also been observed from this study that in low incidence countries the TB/COVID-19 patients die because of co-morbidities and advanced age, but this does not exclude that in intermediate/low income countries we will not observe in the future important mortality occurred in young patients with risk factors (alcohol, smoke, HIV) and advanced drug resistant TB (DR-TB), eventually undergoing several rounds of treatment. An observational study from China reported that persons with active or latent TB have increased susceptibility for SARS-CoV-2 infection associated with rapid progression and severe involvement [25]. Another study also reported that long term impact of SARS-CoV-2 could activate latent form of TB [28]. Various hypothesis have been postulated like reactivation of dormant lesions of TB due to lung inflammation caused by virus, flaring of tubercular lesions due to co-infection with viruses like influenza A triggering type 1 interferon signaling pathway that is also important for mycobacterial growth and activation of mesenchymal stem cell mediated defense mechanism (CD271+BMMSCs) $[28,29]$. Other common pathogenetic mechanisms for coinfection have also been postulated [30-32]. SARS-CoV-2 infection could predispose for TB infection by initiating an aggressive inflammation by increasing cytokines secretion such as interleukin-1 $\beta$ (IL-1 $\beta$ ), interferon- $\gamma$ (IFN- $\gamma$ ), tumour necrosis factor- $\alpha$
(TNF- $\alpha$ ), interleukin-2 (IL-2), interleukin-4 (IL-4) and interleukin10 (IL-10) causing increase in their plasma levels leading to a socalled cytokine storm responsible for severity; immune system hyper-reaction as observed for influenza virus and decreased host resistance due to mutations in the IFN- $\gamma$ and IL-12 signaling pathways. Tocilizumab (TCZ) has been widely used for treatment of severe COVID-19 and patients need to be screened for latent TB. Treatment is to be initiated if tests for latent TB are positive as TCZ competes with IL-6 at receptor level leading to decreased anti-mycobacterial activity by reducing macrophage and cytotoxic cell differentiation. Possibility of reactivation of TB should always be kept in mind. Data regarding this is scarce so benefit of drug should not be compromised during management of severe COVID-19 patients. Tuberculin skin test (TST) and interferongamma release assays (IGRAs), are widely used for latent TB screening. There can be false negative findings in case of immunosuppression. High C-reactive protein (CRP) and lymphopenia in SARS-CoV-2 can lead to false negative tests [33]. Therefore, possibility of latent TB may remain undetected. However, all of these findings need to be validated with robust evidence.

\section{TB and COVID-19 coinfection: experience from our centre}

We are sharing our own experience as we recently encountered two cases in our centre. First case (Case \#1) was 76 years old hypertensive female patient with a 1.5 months history of low-grade intermittent fever, non-productive cough and decreased appetite with an eventual weight loss of $4 \mathrm{~kg}$. She had worsening of symptoms five days prior to presentation with high grade fever followed by breathlessness after 2 days. She was having acute hypoxemic respiratory failure and acute kidney injury. A CT chest was done and showed left lower lobe consolidation with bilateral ground glassing as shown in Figure 1 A-C. Provisional diagnosis of community acquired pneumonia was established initially. Antibiotics were initiated along with oxygen inhalation via face mask and also positive airway pressure intermittently to reduce work of breathing. While undergoing evaluation, her sputum for GeneXpert Ultra revealed Mycobacterium tuberculosis complex detected with indeterminate Rifampicin sensitivity although the direct smear examination for acid fast bacilli was negative. We initiated her on antitubercular treatment on preliminary basis considering clinico-radiological presentation and high specificity of genotypic test, pending culture reports. In view of history of contact with COVID-19 positive relative and presence of acute renal failure, her RT PCR test was sent that was also positive for COVID-19. Although the false negatives in this test are frequent, it rarely presents as false positive and has a very high specificity for COVID-19. We started the treatment for COVID-19 along with anti-tubercular therapy.

Another case (Case \#2) was 42 years old diabetic female presented with high grade fever, myalgia, cough, sore throat and chest tightness for 10 days. Subsequently, she developed breathlessness 4 days prior to her presentation. There was prior history of antitubercular therapy intake for 9 months without any interruption for right pleuro-pulmonary TB that was diagnosed fifteen years back. Chest CT scan revealed multiple patchy and ground glass density shadows in both lungs as shown in Figure 1 D-F. Right pleural thickening with adhesions and partial calcification along with subpulmonary lesions, were also observed. She was tested IGRA positive for TB infection but was AFB smear negative. M. tuberculosis complex was not detected on GeneXpert and preliminary 
Table 1. Characteristic of studies reporting TB and COVID-19 coinfection.

Author Number of cases reported Main findings of the study
with TB and COVID-19
coinfection

Tadolini [21] $\quad 49$

(Multicentre study) Male-40 (81.6\%)

Female-9 (18.4\%)
Active TB-42 (85.7\%); Post TB sequelae-7 (14.3\%)

Pulmonary TB-48 (97.9\%)

Extra-pulmonary TB-13 (26.5\%)DR-TB - 8 (16.3\%)

Confirmed COVID-19- 46 (93.9\%)

Diagnosis by computed tomography thorax- 3 (6.1\%)

BCG vaccination- $19 / 30(63.3 \%)$

HIV infection-6/48 (12.5\%)

TB before COVID-19 diagnosis-26 (53.0\%)

TB after COVID-19 diagnosis-14 (28.5\%)

Diagnosis of TB and COVID-19 simultaneously or within 7 days-9 (18.3\%)

Diagnosis of COVID-19 in patients with TB sequelae-7 (14.3\%)

Health workers involved -2 (4.1\%)

Mortality more in patients with TB sequelae due to older age group and associated comorbidities

\begin{tabular}{|c|c|c|}
\hline $\begin{array}{l}\text { Stochino [22] } \\
\text { (Single centre study) }\end{array}$ & $\begin{array}{l}20 \\
\text { Male-12 (60\%) } \\
\text { Female-8 (40\%) }\end{array}$ & $\begin{array}{l}\text { Pulmonary TB- } 19 \text { (95\%) } \\
\text { Associated Extra-pulmonary involvement- 3/19 (15.8\%) } \\
\text { Exclusive Extra-pulmonary involvement- } 1 \\
\text { TB was diagnosed using CBNAAT- 18(95\%) } \\
\text { Diagnosis confirmed by culture-14/18 (77.8\%) } \\
\text { DR-TB- } 5 \\
\text { BCG vaccination-3 } \\
\text { Mortality-1 }\end{array}$ \\
\hline $\begin{array}{l}\text { He [23] } \\
\text { (Single centre study) }\end{array}$ & $\begin{array}{l}3 \\
\text { All males }\end{array}$ & $\begin{array}{l}\text { Past history of pulmonary TB taking anti-tubercular drug treatment in first and second case } \\
\text { Untreated TB for } 50 \text { years- third case } \\
\text { All were treated }\end{array}$ \\
\hline $\begin{array}{l}\text { Liu [24] } \\
\text { (Single centre study) }\end{array}$ & $\begin{array}{l}3 \\
\text { All males }\end{array}$ & $\begin{array}{l}1^{\text {st }} \text { Case- Latent TB (IGRA+, Sputum for AFB -ve, Culture -ve) with COVID-19 } \\
2^{\text {nd }} \text { Case- Active Drug Resistant TB already on second line drugs with COVID-19 (IGRA+, sputum } \\
\text { for AFB smear }+ \text {, Culture }+ \text { ) } \\
\text { Sputum for COVID-19 also positive } \\
3^{\text {rd }} \text { case- Previous history of TB with IGRA + Diagnosis of COVID-19 with features of ARDS } 3 \text { days } \\
\text { after admission } \\
\text { All were treated }\end{array}$ \\
\hline $\begin{array}{l}\text { Chen [25] } \\
\text { (Multicentre study) }\end{array}$ & $\begin{array}{l}13 \\
\text { for these patients with coinfection) }\end{array}$ & $\begin{array}{l}\text { Latent TB infection (IGRA +ve) with COVID-19:-13 } \\
\text { Active TB-3 with drug resistance in 1, Recovered TB-, Old TB calcification-3, IGRA +ve-2 } \\
\text { Latent TB infection with severe/critical COVID-19:-7 } \\
\text { Patients developed ARDS-5/7 } \\
\text { IGRA + in ARDS subset-4/5 } \\
\text { TB co-infection with disease severity (severe/critical 78\% us mild/moderate cases 22\%; } \mathrm{p}=0.0049 \text { ) } \\
\text { Infection to development of symptoms (MTB+COVID-19: } 6.5 \pm 4.2 \text { days vs COVID-19: } 8.9 \pm 5.2 \\
\text { days; } \mathrm{p}=0.073 \text { ) } \\
\text { Symptom development to severe disease (MTB+COVID-19: } 3.4 \pm 2.0 \text { days vs COVID-19: } 7.5 \pm 0.5 \\
\quad \text { days; } \mathrm{p}=0.075 \text { ) }\end{array}$ \\
\hline $\begin{array}{l}\text { Motta [26] } \\
\text { (Multicentre study) }\end{array}$ & $\begin{array}{l}69 \\
\text { Cohort A-49 (71.1\%) } \\
\text { Cohort B-20 (28.9\%) } \\
\text { Male-52 (75.4\%) } \\
\text { Female-17 (24.6\%) }\end{array}$ & $\begin{array}{l}\text { Migrants: } 43 / 69 \\
\text { Cohort A: } 26 / 49(53.1 \%) \\
\text { Cohort B: } 17 / 20(85.0 \%) \\
\text { Mortality: } 8 / 69(11.6 \%) \\
\text { Cohort A: } 7(14.3 \%) \\
\text { Cohort B: } 1 \text { (5\%) } \\
\text { TB diagnosed before COVID-19- } 6(8.7 \%) \\
\text { Post TB sequelae with COVID-19-1(1.5\%) } \\
\text { TB and COVID-19 diagnosed simultaneously-1(1.5\%) } \\
\text { Male/Female- } 7(14.3 \%) / 1(5 \%) \\
\text { BCG vaccination-3 (37.5\%) } \\
\text { Pan-susceptible strains-7 (14.3\%) } \\
\text { Mycobacterium bovis intrinsically resistant to pyrazinamide-1 } \\
\text { Migrants were younger and having lesser comorbidities as well as mortality compared to natives }\end{array}$ \\
\hline $\begin{array}{l}\text { Kumar [27] } \\
\text { (Single centre study) }\end{array}$ & $\begin{array}{l}\text { Single case } \\
\text { Male }\end{array}$ & $\begin{array}{l}\text { Low grade fever, cough with expectoration and breathlessness since } 1.5 \text { months } \\
\text { COVID-19 RT-PCR + for severe acute respiratory infection } \\
\text { Empirical anti-tubercular therapy started on non-resolving symptoms } \\
\text { Died due to progressive illness } \\
\text { Diagnosis of TB confirmed post mortem by positive CBNAAT }\end{array}$ \\
\hline
\end{tabular}

BCG, Bacillus Calmette-Guerin; CBNAAT, cartridge based nucleic acid amplification test; COVID-19, coronavirus disease 2019; DR-TB, drug-resistant tuberculosis; HIV, human immunodeficiency virus; IGRA, interferon-gamma release assay; RT-PCR, reverse transcriptase- polymerase chain reaction; TB, tuberculosis. 
Mycobacterium Growth Indicator Tube (MGIT) culture was also found to be negative subsequently. Nasopharyngeal swab tested positive for COVID-19 on second day after admission. She developed moderately severe hypoxemic respiratory failure $\left(\mathrm{PaO}_{2} / \mathrm{FiO}_{2}\right.$ $180 \mathrm{mmHg}$ ). She was given oxygen inhalation via face mask with reservoir bag but her oxygenation could not be maintained. Positive airway pressure was applied subsequently to maintain oxygenation and also to reduce work of breathing. She was treated with hydroxychloroquine, azithromycin and methylprednisolone ( $80 \mathrm{mg}$ daily). As the TB lesions in his right lung and pleura were stable without anti-tubercular treatment, and COVID-19 symptoms had resolved, she was discharged after 21 days. These cases highlight the possibility of coexisting infections in TB endemic countries like India that are also facing the COVID-19 pandemic.

\section{TB and COVID-19:}

\section{association causal or just coincidental?}

The coexistence might be just a coincidence but convincing evidence required to establish any causal relationship [34]. Although diagnosis of COVID-19 was established in TB patients at different times in all of these studies, it seems to be doubtful as most of the cases might already have TB before diagnosis due to chronic or insidious onset. This association might be due to unmasking of subtle active TB infection by COVID-19, establishment of diagnosis only when TB patients had superimposed acute onset symptoms of COVID-19, use of immunosuppressant drugs and insufficient infection control practices compounded by a higher susceptibility of TB cases. Therefore, it becomes major concern that diagnosis of TB might be missed or delayed due to overlapping clinical and radiological features leading to unfavourable outcome and enhanced transmission of infection in community. Coinfection of TB with COVID19 cannot be underestimated as every country is already affected with TB whereas COVID-19 is spreading rapidly across many countries. Epidemic of TB also including DR-TB with HIV co-infection is also prevailing in many countries. It can be anticipated that more number of cases will evolve over coming period with the most deadly combination of DR-TB, HIV and COVID-19. Possible drug interactions can be expected with novel COVID-19 drugs based on past experience with anti-tubercular therapy like additive hepatotoxicity (remdisivir/favipiravir/TCZ and rifampin/isoniazid/pyrazinamide), hyperuricemia (favipiravir and pyrazinamide), thrombocytopenia (TCZ and rifampin), QT prolongation (hydroxychloroquine/favipiravir and fluoroquinolones) and skin rash (ivermectin and any antitubercular drugs). Clinicians should remain vigilant for coinfection of TB with COVID-19 during the ongoing pandemic, as therapeutic strategies need to be determined accordingly to improve outcome.

\section{Challenges to combat tuberculosis in ongoing COVID-19 pandemic}

Social distancing is one of important preventive measure used by public health officials to mitigate spread of ongoing COVID-19 pandemic, especially to allow healthcare systems to prepare and ensure proper allocation of resources. Most of the countries have imposed nationwide lockdown to flatten the curve of pandemic. It
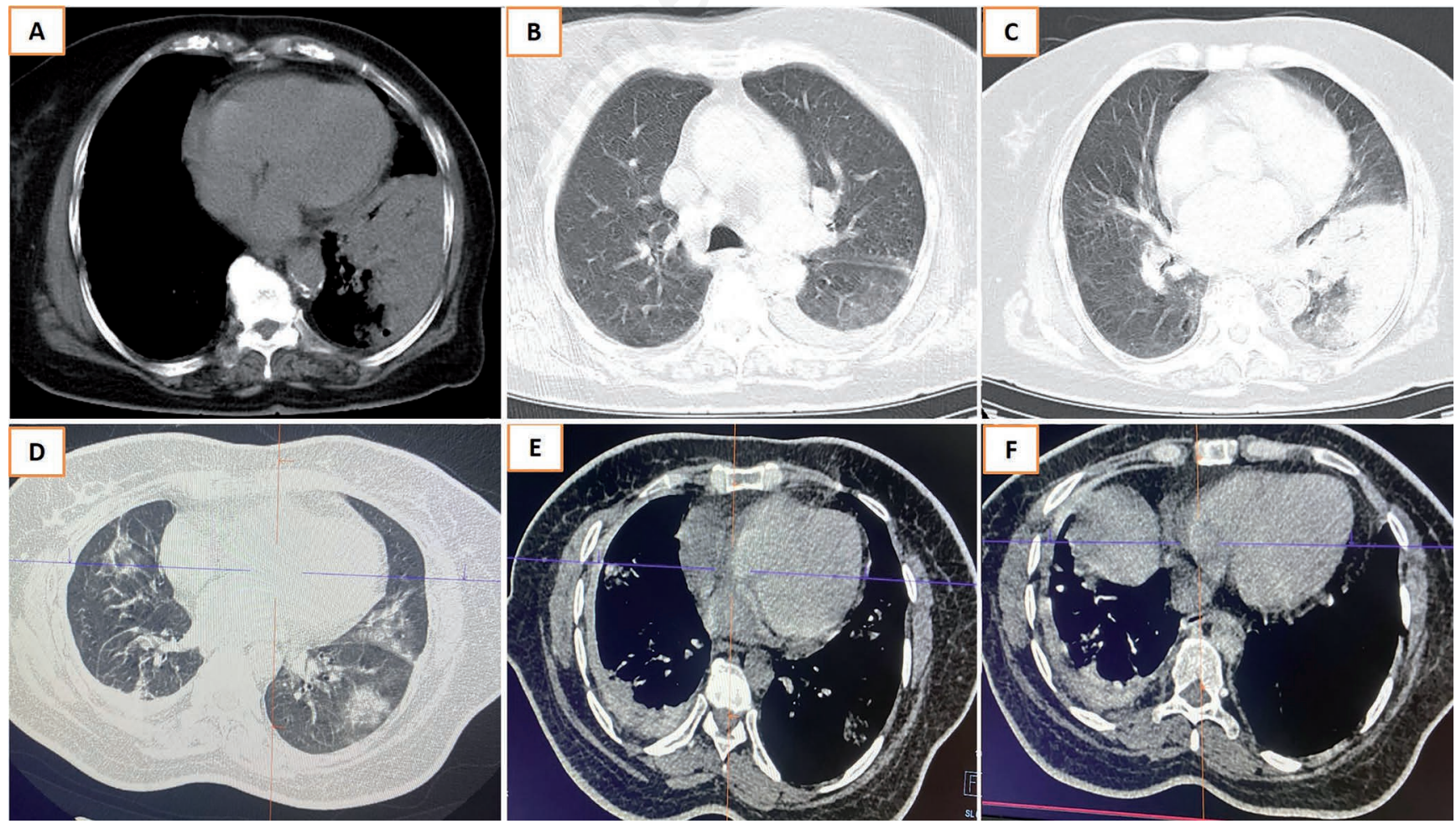

Figure 1. Computed tomography thorax findings of Case \#1 (A-C) showing bilateral diffuse ground glassing and left lower lobe dense consolidation having air bronchogram with underlying effusion; and of Case \#2 (D-F) showing multiple patchy and ground glass density shadows in both lungs with right pleural thickening with adhesions and partial calcification along with sub-pulmonary lesions. 
becomes quite challenging to ensure streamlined delivery of routine health care by programmatic services for other chronic respiratory diseases like TB and HIV due to restricted mobility. Expansion of health services is not possible within short span due to acute health burden. It has been known from past experience with previous viral epidemics that lack of streamlined policy, reduced access to routine health care services including medicines, vaccines and diagnostics, lack of community education, less public engagement due to social stigma associated with myths created by media hype and apprehension even among health care workers regarding novel disease leading to sub-optimal health care services, were major factors responsible for mortality due to TB. A study from West Africa reported 50\%-60\% decrease in the TB services with doubling of mortality rate during Ebola virus outbreak in 2014-2015 [35]. These issues are likely to prevail in this era of ongoing COVID-19 pandemic. It is expected that we are going to witness unfavourable outcome due to possibilities of delay in management of TB in coming months. Various issues that need to be encountered are acute shortage due to diversion of staffs already engaged under national health programmes related to TB, HIV and malaria; priority to diagnose COVID-19 rather than TB by laboratories leading to diagnostic delay of latter; decreased active surveillance for TB; use of DR-TB management centres and critical care services for admitting patients with COVID-19 leading to shortage of beds and ventilators for critically ill TB patients; depletion of drug stocks as well as delay in procurement chain due to disruption of transporting network and manufacturing capacity; interruption of Directy Observed Treatment Short-course (DOTS) services to TB patients with or without HIV and de-prioritization of contact tracing as well as preventive therapy due to restricted mobility created by infection control measures [36-38]. Another issue is that patients with TB stay in close proximity to others in overcrowded areas such as refugee camps, migrants or prisoners, are at higher risk to suffer from COVID-19 as social distancing is practically not possible. All these factors are responsible for delay in TB diagnosis leading to spike in incidence rate and increased transmission of disease.

Rapid spread of COVID-19 with serious illness has overwhelmed efficient health care services of western countries like USA and European region. The situation is more alarming for high burden countries like South Africa, China and India. A modelling analysis released by STOP TB partnership focused on three high burden countries - India, Kenya and Ukraine and predicted that under a 3 month lockdown and a protracted 10 month restoration of services, the world could see an additional 6.3 million TB cases between 2020 and 2025 with an additional 1.4 million TB deaths during the same period [39]. The impact of lockdown can be perceived even in India as only 34,566 TB patients were notified during last three weeks of nationwide lockdown in comparison to 114,460 patients in early March, 2020 (pre-lockdown phase) [40]. The drop was due to the fact that most of the private hospitals and clinics remained non-functional as $70 \%$ of TB patients depend upon private health sector. Patients could not reach DOTS centre to get regular stock of TB drugs, nor did they have access to their regular hospital visits while experiencing adverse events. Health care workers are also unable to step out in the field and provide essential services like monitoring of adverse events and counselling. Even if the drug supply is ensured, initial months are quite crucial as new patients encounter several adverse events like nausea, vomiting, gastritis and psychosis that can create breach in adherence leading to poor outcome [39]. Possibility of emergence of drug resistance could not be ruled out due to malabsorption of drugs from the gut.

\section{Measures to combat tuberculosis in ongoing COVID-19 pandemic}

It becomes essential that national TB programmes should remain operational and that people have easy access to diagnostic services, treatments and support services for TB during this era of COVID-19 pandemic [5]. All countries, especially those with high burden of TB, must procure and maintain intact supply, to ensure adequate supplies of TB medications with timely ordering of new drugs to avoid stock-outs. Principles of DOTS that involve regular supervision might become infeasible to implement, particularly if national quarantine measures are put in place. TB programmes should consider what options can be implemented within a short period of time particularly to needy ones. TB programmes should adopt alternative strategy in place to continue to support patients on TB treatment by ensuring adherence, monitoring of side effects from the medications and providing psycho-social support. Selfadministered therapy can be adopted by provision of drugs for atleast one month in advance preferably from nearest DOTS centre or can be provided even at home $[5,41,42]$. Other measures like focus on triaging with avoidance of routine outpatient department visits and admission of only critically ill patients, task-shifting of health care workers, active surveillance for both diseases, diversion of funds for TB in addition to COVID-19 with aid of donor support having public-private partnership (Global Fund, UNITAID and others), optimal distribution of drug stocks, personal protective equipments and respirators for health care workers, involvement of accredited private laboratories for testing, promotion of virtual and long distance events in place of conferences for information, education and communication to create health awareness among community as well as health care workers and utilization of digital platforms such as virtual OPD or Video-Observed Therapy (VOT), smart pill boxes and other mobile phone-supported adherence strategies in the form of tele-consult, such as 99DOTS [5,42]. The frontline health care workers need to be well prepared by undergoing formal training to provide care for their patients with TB and COVID-19. Community volunteers may be appointed for awareness raising, prevention and early notification for TB in addition to COVID-19. All these measures emphasize on patient centric care that may favour better outcome even in these adverse conditions. Drug resistant tuberculosis (DR-TB) requires special attention and all longer or shorter oral regimens should be prioritized over conventional regimens containing injectables, creating less burden on DOTS provider. Strategies like contact tracing, active surveillance and infection control adopted for curbing pandemics of COVID-19 can complement programmes focussing on TB elimination favouring better outcome.

\section{Global response to combat tuberculosis in ongoing COVID-19 pandemic}

The whole world is focussed on battling COVID-19 pandemic with less concern for other non-COVID-19 illnesses like TB. Forty percent of NTPs across 20 high burden countries reported that they have utilized TB facilities for COVID-19 management and plan to utilize completely if it becomes more widespread [43]. This can create another silent pandemic of TB in coming time. China and South Africa have utilized their existing resources and also expanded their infrastructure to cope up with ongoing crisis. China 
promoted digital medicine to make medical care easily accessible to patients regardless of distance [44]. Health care system of South Africa is already fighting deadly syndemic of TB including drugresistant cases and HIV [45]. South Africa has established mobile health clinics involving primary health care for screening of COVID-19 and ensuring adherence to treatment of TB and HIV patients. The country has implemented door to door testing nationwide by mobilising more than 28,000 health workers to offer routine health care at doorstep [46]. West African countries have developed structured health infrastructure to certain extent that was established with support of Global Fund to Fight AIDS, Tuberculosis and Malaria (GFATM) during Ebola outbreak. This can provide added advantage to combat COVID-19 pandemic as preventive measures can complement control of both diseases. However, these countries still require further preparedness to combat COVID-19 and TB/HIV together which is considered to be a herculean task [47].

\section{Tuberculosis control in COVID-19 pandemic: Indian scenario}

Reallocation of resources is quite challenging for high burden countries for TB like India, as several hurdles exist like significant burden of undiagnosed TB patients including drug resistant ones, coinfection with HIV, priority to treat DR-TB patients with regimens containing newer drugs, non-uniform distribution of health care with poor quality in public sector and low health expenditure $(1.4 \%$ to $1.5 \%$ of gross domestic product) [39,48,49]. Malnutrition, poverty, forced migration of daily wage workers and labourers during abrupt implementation of lockdown, overcrowding in slums and unregistered colonies as well as diverse religious as well as sociocultural norms are other prevailing factors, creating hindrance to containment measures adopted for controlling outbreak. Malnutrition accounts for 7 hundred thousands TB cases in India and can further increase because of the lockdown's impact on livelihoods of migrant workers [39]. Forty percent of Indian population already has latent TB and can transform into active form due to malnutrition. There is high chance of transmission of infection to family members from undiagnosed TB cases particularly among population residing in slums with less space (24\% of total Indian population according to World Bank), facilitating overcrowding during lockdown. Many TB hospitals in Western India run in a partnership with international non-profit organization Medecins Sans Frontieres (MSF) that has introduced measures to prevent transmission of infection such as alternate days Out Patient Department (OPD) services, waiting area in open places like garden, provision of masks and handwashing corners, volunteers for co-ordination of services, psychosocial counselling, simultaneous screening by health workers for COVID-19 as well as TB and promotion of tele-consultation and virtual OPD [39]. India is also planning to impose ban on export of anti-tubercular drugs for atleast one year in order to maintain stock [50]. Pharmaceutical companies are facing difficulties to manufacture new stocks due to lack of raw material, transportation facilities and man-power. India has also provided directives on $26^{\text {th }}$ and $27^{\text {th }}$ March 2020 to ensure uninterrupted diagnosis and treatment of TB patients, including steps like supplying drugs for one month in advance [41,42]. Despite of this, there has been a decline in TB notification for March to April 2020 by $51 \%$ as compared to previous year for same period [51]. As compared with average 8000 TB notification each day last year, there has been decline to 2000 TB cases daily during lockdown period. Diagnosis of new TB cases has seen a dramatic drop during extended phase of lockdown, by almost $80 \%$ and $90 \%$ in public and private sectors respectively by the end of April 2020, when compared to same month the previous year [39]. This could be due to significant heterogeneity existing even across several states of India regarding response to pandemic based on existing health infrastructure and demographic profile. Considering this issue, the Government of India has recently released newer directives on $27^{\text {th }}$ April 2020 in order to maintain and allocate TB services based on local needs and specified regional zones (red, orange and green) [52]. Few states like Tamil Nadu and Kerala have done exceptionally well not only in combating COVID-19 pandemic, but also ensured by providing drugs for two months in advance, weekly digital interaction directly with state headquarters, teleconsultation by health care workers, provision of personal protective equipments and use of two wheelers to provide home delivery [51]. The reason for that state of Kerala has better quality of health care and adequate funding even at public sector and also has experience of successful combat with a Nipah virus outbreak 2 years ago. Focusing solely on COVID-19 can lead to damage of fragile gains that have been achieved for elimination of TB under END TB strategy by WHO. It has been predicted that the global TB incidence and deaths in 2021 would increase to levels last seen between 2013 and 2016, implying a setback of atleast 58 years in the fight against TB [39]. Therefore, it is very essential that people continued to be screened and tested for TB in addition to COVID-19 according to the national guidelines. Investment should be directed towards universal health coverage and social benefits.

\section{Role of Bacille Calmette-Guérin vaccine}

Bacille Calmette-Guérin (BCG) is the only vaccine available for TB that prevents dissemination, whereas for COVID-19 vaccines are still under development. A study from New York Institute of Technology (NYIT) predicted that there could be a possible link between BCG vaccination and its protective effects against COVID-19, by correlating the global spread of COVID-19 with the data from the world atlas that shows nation wise BCG vaccine coverage [53]. Countries such as India, China and Japan with a policy of universal BCG vaccination have had a lower number of cases than those in Spain, France, and Switzerland that discontinued their universal vaccine policies due to comparatively low risk for developing $M$. bovis infection as well as the proven variable effectiveness in preventing adult TB, while countries such as the United States, Italy and Netherlands have yet to adopt universal policies for similar reasons. This, study also tries to explain the anomalous case of Iran where the universal immunization programme for BCG was only recently started in 1984, unlike China and India, whose programs go back to the late 1940s. Hence, most deaths in Iran are of the elderly who did not receive any immunization under the program [53]. The possible explanation on how BCG vaccination can provide immunity against the COVID-19 is that this vaccine has been shown to produce positive heterologous or non-specific immune effects leading to improved response against other non-mycobacterial pathogens. In a study conducted by Mathurin et al., the BCG vaccinated mice infected with the vaccinia virus were protected by increased production of IFN- $Y$ by CD4+ cells [54]. BCG vaccination has been shown to provide broad protection against viral infections and sepsis [55], raising the possibility that the protective effect of the vaccine might not be 
directly related to actions on COVID-19 but on associated cooccurring infections or sepsis. Few epidemiological studies also supported protective role of BCG vaccination against COVID-19 infection [56-58]. BCG revaccination practices, particularly in elderly age group, may provide additional protection against severe COVID-19. Even though the data might suggest the positive role of BCG immunization against COVID-19 we must take into consideration that these are just a mere speculation with limited evidence and hence, not a proven entity. All these findings were strongly criticized by the researchers from the McGill International TB Centre, Montreal, with context to methodology adopted for analysis and the global extent of COVID-19 spreading at the time of study [59]. It was stated that there could be possible harm in citing that BCG may boost immunity in individuals and provide nonspecific protection against COVID-19 without any clear evidence. In countries such as India, the wide BCG coverage offered by the universal vaccination policy may create a false sense of assurance leading to inaction. It was also brought to attention that India had a fewer number of cases at the time NYIT analysis as compared to the current scenario, hence such speculations should not be made unless there is concrete evidence. Another major drawback of the study was that the countries that were selected were arbitrary. Even Australia and Germany have a much lower morbidity and mortality rate despite having no universal BCG immunization policy, hence BCG vaccination solely cannot be the answer. Also, health systems' capacity and infrastructure, are all confounding factors which have not been taken into consideration. The innate immunity of people in low-income countries exposed to dust and grime also needs to be factored in [59]. Whether BCG will be effective remains unknown: findings from the ecological studies suggesting less COVID-19 in countries with routine BCG immunization are weak evidence because they are based on population rather than individual data and are prone to confounding [59]. A recent study on large population-based cohort of Israeli adults aged 35 to 41 years reported that BCG vaccination in childhood was associated with a similar rate of positive test results for SARS-CoV-2 compared with no vaccination ( $11.7 \%$ vs $10.4 \%$; difference, $1.3 \%$; $95 \%$ $\mathrm{CI},-0.3 \%$ to $2.9 \% ; \mathrm{p}=0.09$ ) [60]. Due to small number of severe cases, no conclusion about the association between BCG status and severity of disease can be reached. All these findings need to be validated with robust trials. Few trials from different countries are under progress to settle this conflict. A randomized control trail BRACE trial is undergoing recently in Australia in which 4170 healthcare workers are being enrolled to evaluate the efficacy of BCG vaccination in providing immunity against COVID-19 [61]. Similarly, a trial in Netherlands aims to evaluate the efficacy of BCG vaccination in reducing Health Care Workers Absenteeism in SARS-CoV-2 [62]. An upcoming trial by the Serum institute of India is currently under consideration to test the efficacy of BCG in high-risk COVID-19 groups in Maharashtra, India [63]. Although inconclusive, weather and temperatures may also play a part in the spread and containment of an epidemic that needs to be researched too, since most tropical countries have had low caseloads [64].

\section{Future perspectives}

Whole world is currently facing intense panic or anxiety, social disruption and scientific uncertainty and this has led to rapid policy response and resource allocation to combat COVID-19 rather than for TB. It will be advantageous for mankind to curb existing pan- demic of TB along with ongoing COVID-19 for curbing the coexisting pandemics. There is utmost requirement for further investment and research on newer vaccines as well as drugs for both diseases with aim to achieve better outcome. There is no doubt that whole world is putting sincere effort to stop ongoing novel COVID-19 pandemic but old silent killer TB which is preventable and treatable, should not be forgotten. This year, World TB day ( $24^{\text {th }}$ march) has also been side-lined. If we show half of the effort to combat COVID19 pandemic at this point of time to eliminate TB, global mortality rate can be reduced remarkably in near future. The literature regarding dynamic interaction between COVID-19 and TB is rapidly expanding. Researchers may be able to establish relationship between two diseases with robust evidence in coming times.

\section{References}

1. Wang C, Horby PW, Gao GF. A novel coronavirus outbreak of global health concern. Lancet 2020;395:470-3.

2. Worldometer [Internet]. Coronavirus outbreak. Accessed on: 5 April 2020. Available from: https://www.worldometers.info/ coronavirus/

3. Gagneux S. Ecology and evolution of Mycobacterium tuberculosis. Nat Rev Microbiol 2018;16:202-13.

4. WHO. Global tuberculosis report 2019. Accessed on: 1 April 2020. Available from: https://www.who.int/tb/publications/global_report/en/

5. WHO. Information Note Tuberculosis and COVID-19. COVID19: Considerations for tuberculosis (TB) care. Geneva: WHO; 2020.

6. Dara M, Sotgiu G, Reichler MR, et al. New diseases and old threats: lessons from tuberculosis for the COVID-19 response. Int J Tuberc Lung Dis 2020;24:544-5.

7. Alagna R, Besozzi G, Codecasa LR, et. al. Celebrating world tuberculosis day at the time of COVID-19. Eur Respir J 2020;55:2000650.

8. Wei-Jie G, Ni Z-Y, Hu Y, et al. for the China Medical Treatment Expert Group for Covid-19. Clinical characteristics of coronavirus disease 2019 in China. N Engl J Med 2020;28:202-13.

9. van Doremalen N, Bushmaker T, Dylan H, et al. Aerosol and surface stability of SARS-CoV-2 compared with SARS-CoV-1. N Engl J Med 2020;382:1564-1567.

10. Millet JP, Moreno A, Fina L, et al. Factors that influence current tuberculosis epidemiology. Eur Spine J 2013;22:539-48.

11. Lai CC, Liu YH, Wang CY, et al. Asymptomatic carrier state, acute respiratory disease, and pneumonia due to severe acute respiratory syndrome coronavirus 2 (SARS-CoV-2): Facts and myths. J Microbiol Immunol Infect 2020;53:402-12

12. Bi Q, Wu Y, Mei S, et al. Epidemiology and transmission of COVID-19 in 391 cases and 1286 of their close contacts in Shenzhen, China: a retrospective cohort study. Lancet Infect Dis 2020. doi: 10.1016/ S1473-3099(20)30287-5.

13. Baud D, Qi X, Nielsen-Saines K, Musso D, Pomar L, Favre G. Real estimates of mortality following COVID-19 infection. Lancet Infect Dis 2020. doi: 10.1016/S1473-3099(20)30195-X

14. WHO. Country and Technical Guidance - Coronavirus disease (COVID-19): Laboratory testing for 2019 novel coronavirus (2019-nCoV) in suspected human cases, 19th March 2020. Geneva: WHO; 2020.

15. Indian Council of Medical Research, Department of Health Research. Strategy for COVID-19 testing in India (vers. 4, April 9, 2020) ICMR; 2020. Available from: https://www.icmr. 
gov.in/pdf/covid/strategy/Strategey_for_COVID19_Test_v4_09 042020.pdf

16. Wang W, Xu Y, Gao R, et al. Detection of SARS-CoV-2 in different types of clinical specimens. JAMA 2020;323:1843-4.

17. Wong CY, Wong KY, Law TSG, et al. Tuberculosis in a SARS outbreak. J Chin Med Assoc 2004;67:579-82.

18. Liu W, Fontanet A, Zhang PH, et al. Pulmonary tuberculosis and SARS, China. Emerg Infect Dis 2006;12:707-9.

19. Low JGH, Lee CC, Leo YS, et al. Severe acute respiratory syndrome and pulmonary tuberculosis. Clin Infect Dis 2004;38:e123-5.

20. Alfaraj SH, Al-Tawfiq JA, Altuwaijri TA, Memish ZA. Middle East respiratory syndrome coronavirus and pulmonary tuberculosis coinfection: Implications for infection control. Intervirology 2017;60:53-5.

21. Tadolini M, Codecasa LR, García-García JM, et al. Active tuberculosis, sequelae and COVID-19 co-infection: first cohort of 49 cases. Eur Respir J 2020;56:2001398.

22. Stochino C, Villa S, Zucchi P, et al. Clinical characteristics of COVID-19 and active tuberculosis co-infection in an Italian reference hospital. Eur Respir J 2020;2001708. doi: 10.1183/ 13993003.01708-2020.

23. He G, Wu J, Shi J, et al. COVID-19 in tuberculosis patients: a report of three cases. J Med Virol 2020. doi:10.1002/jmv.25943.

24. Liu C, Yu Y, Fleming J, et al. Severe COVID-19 cases with a history of active or latent tuberculosis. Int J TB Lung Dis 2020. doi: 10.5588/ijtld.20.0163.

25. Chen Y, Wang Y, Fleming J, et al. Active or latent tuberculosis increases susceptibility to COVID-19 and disease severity. MedRxiv 2020. doi: 10.1101/2020.03.10.20033795.

26. Motta I, Centis R, D'Ambrosio L, et al. Tuberculosis, COVID19 and migrants: preliminary analysis of deaths occurring in 69 patients from two cohorts. Pulmonology 2020;26:233-40.

27. Kumar DR, Bhattacharya DB, Meena DV, et al. COVID-19 and TB co-infection - 'Finishing touch" in perfect recipe to 'severity' or 'death'. J Infect 2020. doi: 10.1016/j.jinf.2020.06.062.

28. Pathak L, Gayan S, Pal B, et al. Corona virus activates a stem cell mediated defense mechanism that accelerates activation of dormant tuberculosis: implications for the COVID-19 pandemic. BioRxiv 2020. doi: 10.1101/2020.05.06.077883.

29. Thomas L. COVID-19 could accelerate activation of dormant tuberculosis (TB). News-Medical [Internet]. May 10th 2020. Available from: https://www.news-medical.net/news/20200510/ COVID-19-could-accelerate-activation-of-dormant-tuberculosis-(TB).aspx.

30. Crisan-Dabija R, Grigorescu C, Pavel C-A, et al. Tuberculosis and COVID-19 in 2020: lessons from the past viral outbreaks and possible future outcomes. MedRxiv 2020. doi: $10.1101 / 2020.04 .28 .20082917 \mathrm{v} 1$

31. Yasri S, Wiwanitkit V. Tuberculosis and novel Wuhan coronavirus infection: Pathological interrelationship. Indian J Tuberc 2020;67:264.

32. Zhang W, Zhao Y, Zhang F, et al. The use of anti-inflammatory drugs in the treatment of people with severe coronavirus disease 2019 (COVID-19): The perspectives of clinical immunologists from China. Clin Immunol 2020;214:108393.

33. Guan WJ, Ni ZY, Hu Y, et al. Clinical characteristics of coronavirus disease 2019 in China. N Engl J Med 2020;382:1708-20.

34. Khurana AK, Aggarwal D. The (in)significance of TB and COVID-19 co-infection. Eur Respir J 2020. doi: 10.1183/ 13993003.02105-2020.

35. Ansumana R, Keitell S, Roberts GMT, et. al. Impact of infectious disease epidemics on tuberculosis diagnostic, manage- ment, and prevention services: experiences and lessons from the 2014-2015 Ebola virus disease outbreak in West Africa. Int J Inf Dis 2017;56:101-4.

36. Wong E. TB, HIV and COVID-19: urgent questions as three epidemics collide. The Conversation [Internet] 24 March 2020. Accessed on: 28 April 2020. Available from: https://theconversation.com/tb-hiv-and-covid-19-urgent-questions-as-three-epidemics-collide-134554

37. Pai M. COVID-19 coronavirus and tuberculosis: We need a damage control plan. Forbes [Internet] 17 March 2020. Accessed on: 30 April 2020. Available from: https://www. forbes.com/sites/madhukarpai/2020/03/17/covid-19-and-tuberculosis-we-need-a-damage-control-plan/

38. Gupta A, Singla R, Caminero JA, et al. Impact of COVID-19 on tuberculosis services in India. Int J Tuberc Lung Dis 2020. doi: 10.5588/ijtld.20.0212.

39. Bedi A, Yadavar S. TB patients badly hit by lockdown- $80 \%$ drop in diagnosis, huge struggle for medicines. The Print [Internet] 30 April 2020. Accessed on: 3 May 2020. Available from: https://theprint.in/health/tb-patients-badly-hit-by-lockdown-80drop-in-diagnosis-huge-struggle-for-medicines/411399/

40. Ghosh A. A silent casualty: Drop in TB reporting, states admit lockdown issues. Indian Express Delhi Edition [Internet] 19 April 2020. Accessed on: 19 April 2020. Available from: https://indianexpress.com/article/india/tuberculosis-cases-dropamid-lockdown-coronavirus-covid-19-6368736/

41. Ministry of Health and Family Welfare, Deputy Director (TB), National Task Force, NTEP. Guidelines for treatment of TB patients under National TB Elimination Program (NTEP), during COVID 19 outbreak. MOHFW; 2020. Accessed on: 12 April 2020.

42. Ministry of Health and Family Welfare, Deputy Director (TB), National Task Force, NTEP. D O letter on TB related services under National TB Elimination Program (NTEP) during countrywide lock-down due to COVID 19. MOHFW; 2020. Accessed on: 12 April 2020.

43. Stop TB partnership [Internet]. We did a rapid assessment: The TB response is heavily impacted by the COVID-19 pandemic. Accessed on: 24 April 2020. Available from: http://www.s toptb.org/news/stories/2020/ns20_014.html

44. Pang Y, Liu Y, Du J, et al. Impact of COVID-19 on tuberculosis control in China. IJTLD 2020; 1-3. http://dx.doi.org/ 10.5588/ijtld.20.0127.

45. Ongole JJ, Rossouw TM, Fourie PB, et al. Sustaining essential healthcare in Africa during the COVID-19 pandemic. Int J Tuberc Lung Dis 2020. doi: 10.5588/ijtld.20.0214.

46. The Washington Post [Internet]. South Africa is hunting down coronavirus with thousands of health workers. Accessed on: 27 April 2020. Available from: https:/www.washingtonpost. com/world/africa/south-africa-is-hunting-down-coronaviruswith-tens-of-thousands-of-health-workers/2020/04/21/ 6511307a-8306-11ea-81a3-9690c9881111_story.html

47. Chakaya J, Binegdie A, Irungu A, et. al. COVID-19 in Africa: preparing for the storm (Correspondence Int $\mathrm{J}$ Tuberc Lung Dis 2020. doi: 10.5588/ijtld.20.0281.

48. The Caravan [Internet]. Amid lockdown, TB patients struggle for diagnosis, treatment; experts predict rise in mortality. Accessed on: 3 May 2020. Available from: https:/caravanmagazine.in/health/amid-lockdown-tb-patients-struggle-fordiagnosis-treatment-experts-predict-rise-in-mortality

49. Scroll.in [Internet]. India's focus on coronavirus leaves TB and HIV patients adrift. Accessed on: 19 April 2020. Available from: https://scroll.in/article/958400/invisible-crisis-tb-and-hivpatients-left-adrift-in-indias-focus-on-coronavirus 
50. Business Standard [Internet]. Lockdown hits production of antiTB drugs, health min seeks to ban export. Accessed on: 30 April 2020. Available from: https://www.business-standard.com/article/economy-policy/lockdown-hits-production-of-anti-tb-drugshealth-min-seeks-to-ban-export-120042101769_1.html

51. Ministry of Health and Family Welfare, Addl. DDG (TB), Central TB Division, GOI, National Task Force, NTEP. Minutes of Vice Chairman meeting held on 20th April regarding NTEP implementation during COVID-19 pandemic. MOHFW; 2020. Accessed on: 26 April 2020.

52. Ministry of Health and Family Welfare, Addl. DDG (TB), Central TB Division, GOI, National Task Force, NTEP. Advisory for States/UTs from Central TB Division. MOHFW; 2020. Accessed on: 2 May 2020.

53. New York institute of Technology [Internet]. TB Vaccine could be a valuable weapon in COVID-19 fight. March 30, 2020. Available from: https://www.nyit.edu/box/features/tb_vaccine_ could_be_a_valuable_weapon_in_covid_19_fight.

54. Mathurin KS, Martens GW, Kornfeld H, Welsh RM. CD4 T-cellmediated heterologous immunity between mycobacteria and poxviruses. J Virol 2009;83:3528-39.

55. Moorlag SJCFM, Arts RJW, Van Crevel R, Netea MG. Non-specific effects of BCG vaccine on viral infections. Clin Microbiol Infect 2019;25:1473-8.

56. Miller A, Reandelar MJ, Fasciglione K, et al. Correlation between universal BCG vaccination policy and reduced morbidity and mortality for COVID-19: an epidemiological study. MedRxiv 2020. doi: 10.1101/2020.03.24.20042937.

57. Hegarty PK, Kamat AM, Zafirakis H, Dinardo A. BCG vaccination may be protective against Covid-19. ResearchGate preprint 2020. doi: 10.13140/RG.2.2.35948.10880.
58. Dayal D, Gupta S. Connecting BCG vaccination and COVID19: Additional data. MedRxiv;2020. doi: https://doi.org/10. 1101/2020.04.07.20053272.

59. Forbes [Internet]. BCG against coronavirus: Less hype and more evidence, please. April 12th 2020. Accessed on: 26 April 2020. Available from: https://www.forbes.com/sites/madhukarpai/ 2020/04/12/bcg-against-coronavirus-less-hype-and-more-evidence-please/\#104fa5076b4f

60. Hamiel U, Kozer E, Youngster I. SARS-CoV-2 rates in BCGvaccinated and unvaccinated young adults. JAMA 2020;323: 2340-1.

61. ClinicalTrials.gov [Internet]. BCG vaccination to reduce the impact of COVID-19 in Australian healthcare workers following coronavirus exposure (BRACE) trial. BCG vaccination to protect healthcare workers against COVID-19. 2020. Available from: https://clinicaltrials.gov/ct2/show/NCT04327206

62. ClinicalTrials.gov [Internet]. Reducing Health Care Workers Absenteeism in COVID-19 Pandemic through BCG Vaccine (BCG-CORONA). 2020. Available from: https://clinicaltrials. gov/ct2/show/NCT04328441

63. The Print [Internet]. BCG vaccine trials to begin in 2 weeks on high-risk COVID-19 groups in Maharashtra. April 20th 2020. Accessed on: 25 April 2020. Available from: https://theprint. in/india/bcg-vaccine-trials-to-begin-in-2-weeks-on-high-riskcovid-19-groups-in-maharashtra/405189/

64. Faust L, Huddart S, MacLean E, Svadzian A. Universal BCG vaccination and protection against COVID-19: critique of an ecological study. April 1, 2020. Available from: https://naturemicrobiologycommunity.nature.com/posts/64892-universal-bcgvaccination-and-protection-against-covid-19-critique-of-anecological-study 